\title{
Objetos virtuais de aprendizagem em saúde: experiência em disciplina de graduação
}

Othon de Carvalho Bastos Filho - othon.filho@ufma.br - DEMED I /UFMA

Bruno do Lago Nascimento - bruno.lago9001.ln@gmail.com - Graduando em Medicina/UFMA

Resumo: Este estudo apresenta considerações acerca da necessidade de mudanças na formação de estudantes, em particular na área da saúde, no sentido de formar profissionais com habilidades e competências para o trabalho em equipe, articulado com os serviços de saúde e comunidade, bem como o desenvolvimento de pensamento crítico e capacidade de aprender. $\mathrm{O}$ objetivo é demonstrar a experiência acadêmica de aquisição de novos conhecimentos e desenvolvimento pessoal durante a construção de Objetos Virtuais de Aprendizagem em disciplina de graduação. Para isso, o Laboratório de Inovações Tecnológicas Aplicadas à Medicina desenvolveu um questionário e aplicou-o com alunos participantes na disciplina Informática Médica. Após o término da referida disciplina, os dados coletados foram estudados. Em análise, constatou-se equilíbrio a partir dos dados obtidos, permitiu-se às equipes de alunos uma revisão dos trabalhos e a abertura para observação e avaliação dos projetos, comparando-os com os de outras equipes, concluiu-se que o método adotado na disciplina traz uma proposta de avaliação contínua e progressiva, fomentando um estado de equilibração e reequilibração dos participantes.

Palavras-chave: Objetos Virtuais de Aprendizagem, Tomada de consciência, Equilibração

\section{Virtual learning objects in health: experience in undergraduate discipline}

\begin{abstract}
This study presents considerations about the need for changes in student training, particularly in the health area, in order to train professionals with skills and competences for teamwork, articulated with health and community services, as well as the development of critical thinking and the ability to learn. The objective is to demonstrate the academic experience of acquiring new knowledge and personal development during the use of Virtual Learning Objects in undergraduate discipline. For this, the Laboratory of Technological Innovations Applied to Medicine developed a questionnaire and applied it with students participating in the discipline Medical Informatics. After completing this discipline, the data collected were studied. In the analysis, a balance was obtained from the data obtained, the teams of students were allowed a review of the works and the opening for observation and evaluation of the projects, comparing them with those of other teams, it was concluded that the adopted method in the discipline brings a proposal of continuous and progressive evaluation, fostering a state of equilibration and rebalancing of participants.
\end{abstract}

Keywords: Learning virtual objects, Awareness Taking, Equilibration 


\section{Introdução}

As Diretrizes Curriculares Nacionais para os cursos de graduação na área da saúde apontam para a necessidade de mudanças no processo de formação dos estudantes. Indicam a necessidade de formar profissionais com habilidades e competências para o trabalho em equipe, para o trabalho articulado com os serviços de saúde e comunidade, bem como o desenvolvimento de pensamento crítico e capacidade de aprender a aprender. Para tanto, faz-se necessário que as Instituições de Ensino repensem seus processos de ensino, discutindo seus projetos políticos pedagógicos e as práticas docentes, a fim de adequá-los a esta necessidade. Considerando-se esta realidade, as ferramentas tecnológicas podem ser vistas como recursos facilitadores para a construção da aprendizagem, uma vez que promovem maior interação, distribuição, agilidade na recuperação e comunicação da informação, nos mais variados contextos.

A crescente inserção de tecnologias baseadas na web na área de educação em saúde revela novas oportunidades de aprendizagem online, de modo flexível e facilitado. Neste cenário, um Objeto Virtual de Aprendizagem (OVA) oferece uma oportunidade para a dinâmica no processo de ensino e aprendizagem em saúde. Esta tecnologia pode ser definida como um recurso digital que pode ser utilizado para o suporte ao ensino, delineado sob uma perspectiva pedagógica e de planejamento integrado ao processo de ensino e aprendizagem.

O conceito de "objetos de aprendizagem" surgiu no final dos anos 1990, mas sua utilização continua significativa com a expansão da educação à distância e o avanço dos recursos tecnológicos. Essa expansão pode ser comprovada através do Censo do Ensino Superior do Ministério da Educação, que registra um crescimento importante no número de matriculados em cursos superiores à distância nos últimos dez anos. No mesmo período, constata-se uma expansão das redes de comunicação, atingindo regiões afastadas dos grandes centros urbanos, permitindo acesso à internet com mais velocidade e qualidade nos mais diversos locais do país.

Os OVAs são cada vez mais utilizados como facilitadores do conhecimento, pois consistem em tecnologias voltadas a estreitar os caminhos do saber, empregados desde a educação básica até em cursos superiores, incluindo os da área da Saúde.

De acordo com Castro-Filho e colaboradores existem duas hipóteses sobre as problemáticas de aprendizagem por parte dos alunos na apropriação de conceitos dentro de cada disciplina: a complexidade própria relativa a esses conceitos e a forma de ensino utilizada pela escola. Para dar conta desses dois aspectos, os objetos de aprendizagem não impõem limites de extensão, porém existe o consenso de que eles devem ter um propósito educacional definido, um elemento que estimule a reflexão do estudante e que sua aplicação não se restrinja a um único contexto.

Diante desde cenário, em decorrência do desenvolvimento das Tecnologias de Informação e Comunicação no processo de ensino e aprendizagem, em particular na 
área da saúde, este trabalho propõe-se a demonstrar a experiência acadêmica de aquisição de novos conhecimentos e desenvolvimento pessoal durante utilização de OVAs em disciplina de graduação.

\section{Referencial Teórico}

Para melhor explicar a interação que ocorre entre os Alunos, sujeito da ação (S) e os OVAs objeto da ação (OB) apontamos na teoria piagetiana, a Tomada de Consciência, como referência para o favorecimento de situações em que o (S) possa fazer a Tomada de Consciência de suas próprias ações e decisões. A ação é um saber fazer, consciente ou não, que conduz ao sucesso ou ao fracasso, que pode ser autônoma em relação à conceituação. Através da(s) tomada(s) de consciência, ou seja, da passagem da assimilação prática (assimilação do objeto a um esquema) a uma assimilação por meio de conceitos (o sujeito vai se apropriando dos mecanismos de ação e delineando as razões que o levam aos resultados) fazendo com que a conceituação passe a dirigir a ação por antecipação, caracterizando uma conduta cognitiva $\gamma$, em que o comportamento do fenômeno ou do objeto da ação passa a ser previsível para o Sistema Cognitivo. (BASTOS FILHO 2006)

[...] a ação, ela só tende para um alvo e ela está satisfeita quando o alvo é atingido. Ela é dominada por aquilo que eu chamaria de êxito. Enquanto que a tomada de consciência comporta mais a compreensão: trata-se de saber como se tem êxito (PIAGET, 1978, p. 127).

Tudo isso é devido ao progresso da regulação da ação (PIAGET, 1977) que, de regulações automatizadas, insuficientes para suscitar uma tomada de consciência adequada, passa a adotar uma regulagem mais ativa (que comporta escolhas mais ou menos intencionais), caracterizada pelo agir em função de uma escolha, fonte normal das tomadas de consciência. Esse progresso é realmente significativo fazendo a ação passar do nível do comportamento material para o da representação, permitindo a incorporação de mais informações, na forma de relações que caracterizam a compreensão.

[...] não há, ação regulada ativamente e, depois, tomada de consciência conceituada e adequada, mas primeiramente uma conceituação heterogênea em relação à ação, depois regulação ativa, e, em seguida, ação ajustada à conceituação, tornando-se, por isso mesmo, consciente. Entretanto, poderá haver uma regulação ativa decorrente de uma conceituação inicialmente estranha à ação efetiva, esses exemplos nos impõem a distinção entre os dois tipos de regulação, automatizada e ativa (PIAGET, 1977, p.16).

Os efeitos resultantes da conceituação sobre a ação se darão devido ao aumento do poder de coordenação de relações (como fazer para alcançar o alvo?) e do seu sistema de conceitos (por que as coisas se passam dessa maneira?), permitindo, assim, uma melhor estruturação do real (neste caso o objeto de conhecimento identificado pelos OVAs.

Este processo de Tomada de Consciência se constitui por uma lei de sucessões, conduzindo o conhecimento do Sujeito $(\mathrm{S})$ da periferia $(\mathrm{P})$ da ação (no ponto de interação) para os mecanismos centrais (C) da ação do sujeito (S), e, ao mesmo tempo, para os mecanismos centrais $\left(\mathrm{C}^{\prime}\right)$ do funcionamento estrutural do objeto $(\mathrm{O})$, 
em suas propriedades intrínsecas, buscando atingir as coordenações internas das ações do S e das propriedades do O (Figura 1). Ou seja, o conhecimento não parte do sujeito (S) e nem do objeto (O), mas sim de suas interações (PIAGET, 1977).

Figura 1: Interação entre o sujeito $(\mathrm{S})$ e o objeto $(\mathrm{O})$, saindo do estado periférico $(\mathrm{P})$ para os centros $(\mathrm{C}$, C').

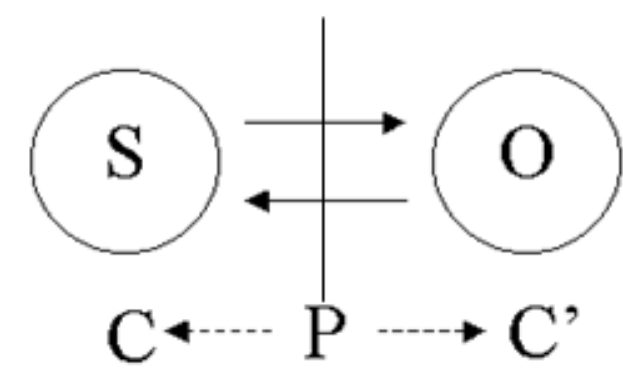

Fonte: Piaget (1977).

\section{Método}

Trata-se de um trabalho descritivo e qualitativo desenvolvido nas seguintes etapas: Definição da pergunta de pesquisa, especificação de critérios de inclusão e exclusão, desenvolvimento de questionário para avaliação qualitativa, aplicação dos critérios de inclusão e exclusão, inclusão de resultados em planilha eletrônica, apresentação de resultados, discussão e considerações.

Os critérios de inclusão foram: o aluno ter realizado matrícula na disciplina eletiva de Informática Médica do curso de graduação em Medicina da Universidade Federal do Maranhão (UFMA), ter realizado no mínimo $70 \%$ das atividades propostas presencialmente e no Ambiente Virtual de Aprendizagem (AVA) do Sistema Integrado de Gestão de Atividades Acadêmicas (SIGAA), ter participado da atividade em grupo de elaboração de projeto e/ou produção de objeto virtual de aprendizagem e, preenchidas as condições anteriores, responder um questionário impresso ou online. Os critérios de exclusão foram o não preenchimento de quaisquer das condições anteriores.

O questionário é uma ferramenta de avaliação desenvolvida pelo Laboratório de Inovações Tecnológicas Aplicadas à Medicina - LITAM para demonstrar a experiência dos discentes em relação ao processo de ensino e aprendizagem dentro da disciplina de Informática Médica. Este meio de avaliação foi aplicado em uma sessão final da referida disciplina, de forma presencial e à distância, por meio do envio das questões via endereço eletrônico. 
As dimensões avaliadas pelo questionário foram: a presença e participação da figura de um monitor da disciplina, discente colaborador do LITAM, desenvolvendo habilidades de docência; avaliação objetiva e subjetiva quanto ao resultado dos trabalhos desenvolvido em equipe dentro AVA SIGAA e apresentados aos demais grupos na sessão final da disciplina; avaliação subjetiva da contribuição dos OVAs apresentados pelos grupos discentes no esclarecimento e/ou desenvolvimento de algum aspecto da atividade acadêmica; avaliação objetiva do SIGAA na organização de atividades acadêmicas em geral e à disciplina específica em particular; fontes adotadas pelos grupos discentes na elaboração do projeto e/ou objeto de aprendizagem; avaliação subjetiva do discente em relação ao método adotado para análise de seu desempenho na disciplina específica; avaliação subjetiva de identificação de conceitos apresentados durante o curso da disciplina específica na atividade da sessão final; avaliação subjetiva da capacidade de discussão de temas abordados durante a disciplina específica ao término das atividades da sessão final; capacidade de identificação de temas abordados na disciplina específica em um cenário prático; avaliação demográfica em relação à idade, gênero, acesso à internet em domicílio.

\section{Resultados e Discussão}

Para análise dos dados coletados neste trabalho, através do questionário proposto, foram desenvolvidos gráficos que apresentam o percentual das respostas discentes, conforme apresentado na Figura 2.

Figura 2: Avaliação de alunos quanto à contribuição dos OVAs no desenvolvimento acadêmico.

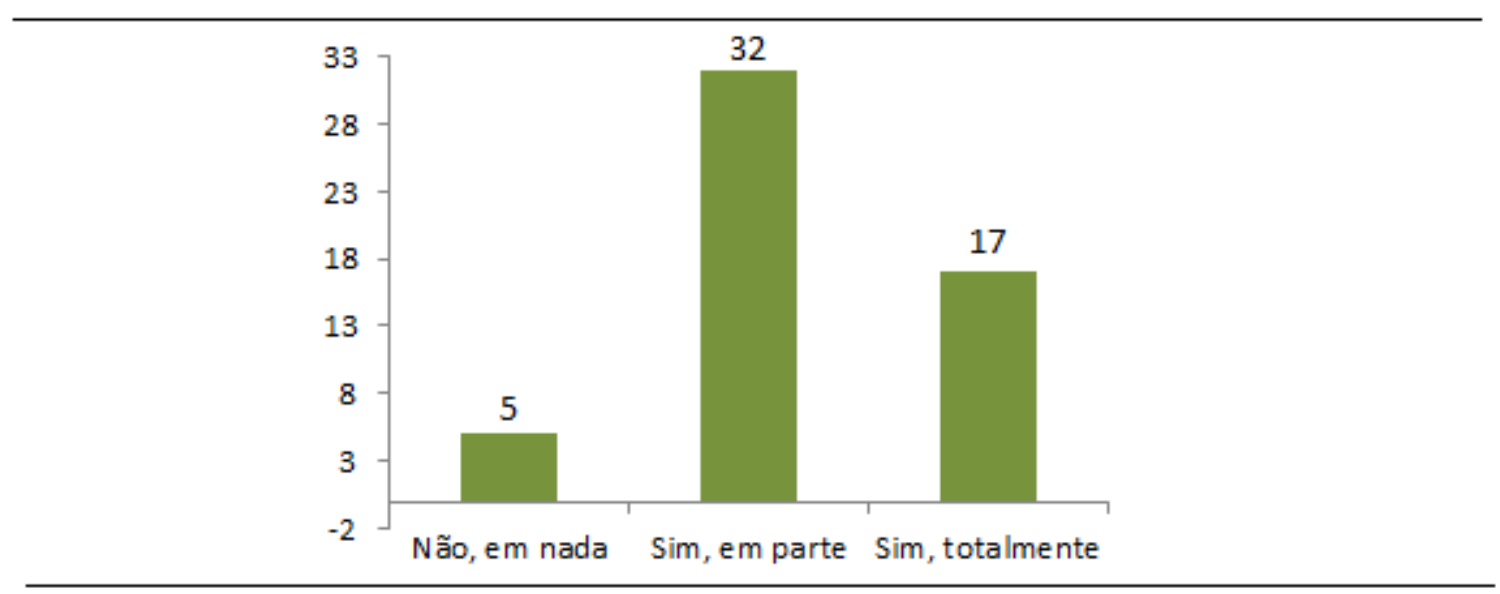

Fonte: Nascimento (2017).

A pesquisa demonstrou que a amostra avaliada é composta de $26(48,14 \%)$ do sexo feminino e $28(51,85 \%)$, do sexo masculino. Para 48 pessoas $(88,8 \%)$, a figura do monitor tem um papel importante durante a ministração das aulas, sendo o número de 6 $(11,1 \%)$ os contrários a esta participação. Em relação à presença dos monitores no processo de ensino-aprendizagem, atuando dinamicamente, 25 (46,2\%) alunos avaliaram a participação da monitoria como boa; o número de avaliações para desempenho ótimo e regular foi coincidente, totalizando 10 (18,5\%); enquanto 9 $(16,6 \%)$ alunos fizeram a classificação da referida participação como muito boa. 
Cada aluno avaliou subjetivamente os trabalhos apresentados pelos demais grupos e classificou-os. Pode-se observar que para $18(33,3 \%)$ alunos os OVAs foram Bons ou Muito bons (de forma semelhante ao item anterior onde houve coincidência entre um item respondido); $9(16,6 \%), 8(14,8 \%)$ e $1(1,8 \%)$ classificaram os OVAs como ótimos, regulares e ruins, respectivamente. Pode-se analisar destes três últimos itens que para um número pequeno $(1,8 \%)$ de alunos os OVAs são classificados como não satisfatórios. A diferença para aqueles que os consideram ótimos e regulares também mostra-se ínfima, sendo um resultado potencialmente esperado dado o número amostral dos participantes da pesquisa.

A figura 2 revela a avaliação que alunos fizeram em relação à importância da apresentação dos OVAs para esclarecimento de dúvidas e/ou desenvolvimento das atividades acadêmicas ao longo do curso de graduação. Para $32(59,2 \%)$ alunos os OVAs contribuíram parcialmente no referido processo; 17 (31,4\%) alunos disseram que os OVAs contribuíram plenamente no aprendizado, enquanto $5(9,2 \%)$ disseram que não houve contribuição. Para este item em particular, observa-se que há nítida diferença entre as respostas apresentadas. Existe um esboço de distribuição normal (Gaussiana) demonstrando números centrais (contribuição parcial dos OVAs no processo de estudo durante a graduação) e valores menores nas extremidades com maior tendência à aprovação.

Em relação à importância dada pelo aluno ao SIGAA como ferramenta de organização acadêmica, 15 (27,7\%), 10 (18,5\%), $11(20,3 \%)$ e 12 (22,2\%) atribuíram notas 10, 9, 8 e 7, respectivamente, alcançando um número geral de $88,8 \%$ de aprovação pelos entrevistados, enquanto o somatório de avaliações com notas inferiores a estas somou $11,1 \%$. Analogamente às notas necessárias para aprovação de discentes em cursos de nível básico ou avançado, pode-se afirmar que houve aprovação do corpo discente participante da pesquisa em relação à importância do SIGAA na organização de materiais e/ou outros OVAs no processo de ensino-aprendizagem.

O item seguinte do questionário faz avaliação objetiva em relação à importância dada pelo aluno ao SIGAA quanto ao seu aprendizado na disciplina de informática médica. Foram atribuídas notas 7 (12 alunos - 22,2\%), 8 (11 alunos - 20,3\%), 9 (10 alunos $18,5 \%)$ e 10 (8 alunos - 14,8\%), respectivamente, somando 75,8\% de aprovação entre os entrevistados, enquanto o somatório das notas inferiores a estas somou 23,9\%, conforme figura 3 . 
Figura 3: Importância dada pelo aluno ao SIGAA no aprendizado específico da disciplina Informática Médica.

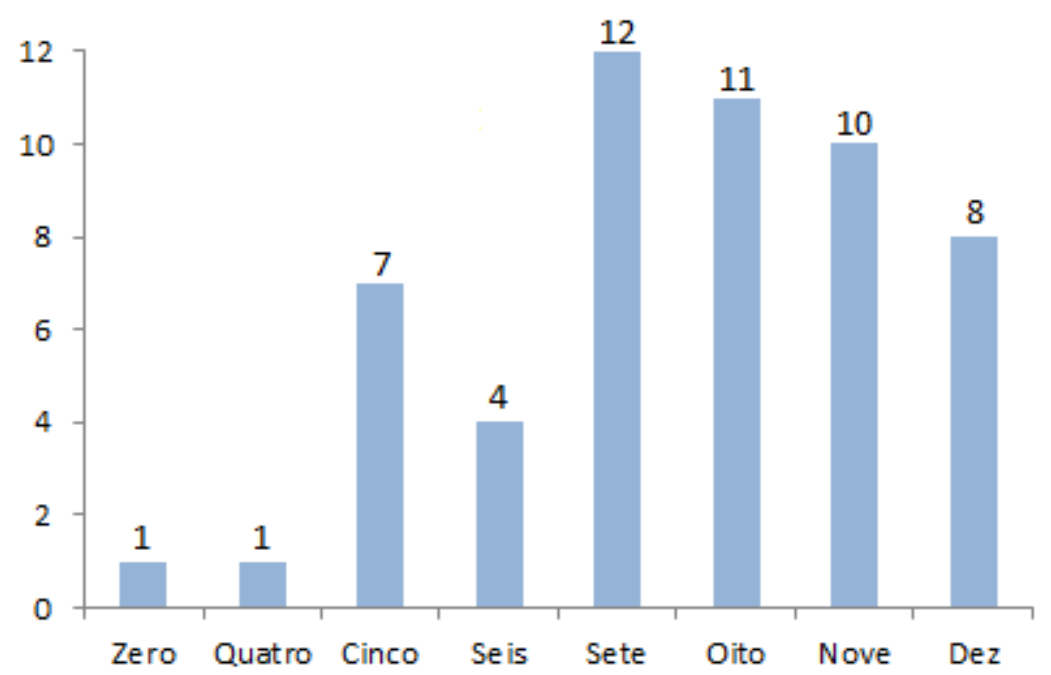

Fonte: Nascimento (2017).

Os alunos foram perguntados sobre as fontes adotadas para desenvolver as atividades propostas durante o curso da disciplina específica. Neste item, os alunos poderiam escolher mais de uma alternativa. Para $48(88,9 \%)$ alunos, o meio mais utilizado de modo geral foi a internet. Livros foram os escolhidos por $15(27,8 \%)$ dos entrevistados. Artigos foram a fonte escolhida por $14(25,9 \%)$ dos discentes. Dentre o grupo pesquisado, 2 acadêmicos $(3,7 \%)$ afirmaram não ter usado nenhuma fonte relacionada no questionário ou não estudaram para desenvolvimento das atividades propostas. Apenas $1(1,9 \%)$ aluno afirmou ter usado revista como meio de pesquisa e $11(20,4 \%)$ acadêmicos afirmaram ter usado outros meios para pesquisas.

Os participantes da pesquisa foram perguntados quanto às suas opiniões sobre o método de avaliação do que aprenderam e produziram no curso de Informática Médica através de atividades online, atividade escrita e apresentação dos OVAs desenvolvidos ou projetos de OVAs. Para 29 (53,7\%) entrevistados, o método foi classificado como bom. Muito bom, foi a classificação adotada por 17 (31,5\%) dos discentes perguntados. Para $2(3,7 \%)$ alunos, o método de avaliação foi classificado como regular. As classificações muito ruim e regular foram a escolha de $2(3,7 \%)$ acadêmicos, enquanto $4(7,4 \%)$ dos entrevistados não responderam a este item.

Um item do questionário aplicado com os alunos, perguntou sobre a capacidade dos acadêmicos, ao final das apresentações das equipes, de reconhecer conceito apresentados ao longo do curso específico. Para 36 (66,7\%) dos entrevistados, a resposta foi "conseguir reconhecer alguns conceitos apresentados". "Todos os conceitos apresentados" foi item referente à escolha de 14 (25,9\%) alunos. O item de "não conseguir reconhecer nenhum conceito apresentado" não foi escolhido por nenhum entrevistado, enquanto $4(7,4 \%)$ dos acadêmicos não responderam a este item. 
Figura 4: Percepção do aluno sobre capacidade de discutir os temas abordados durante todo o curso específico.

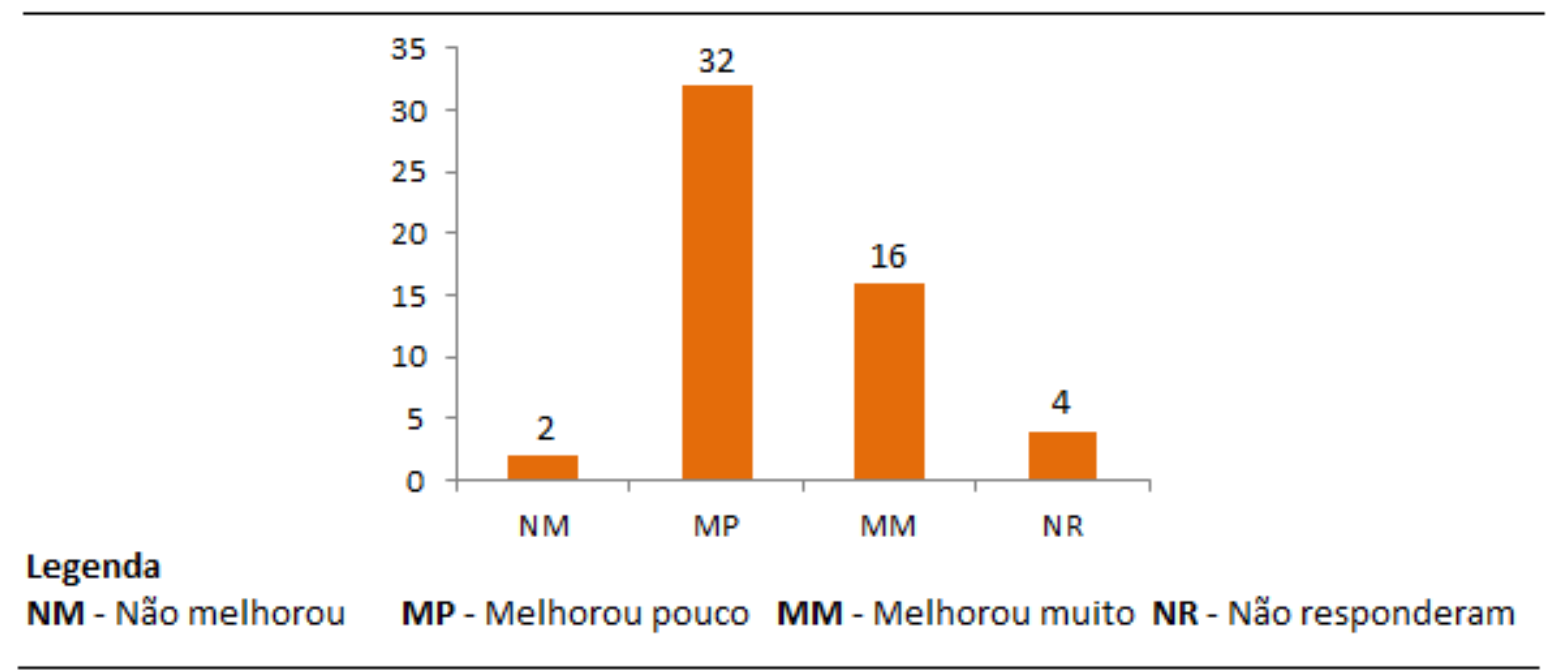

Fonte: Nascimento (2017).

O questionário perguntou também a percepção dos alunos sobre a capacidade de discutir os temas abordados durante todo o curso após a apresentação final da disciplina. Para 2 (3,7\%) acadêmicos a capacidade em questão não melhorou. Para 32 (59,3\%) entrevistados, a capacidade melhorou pouco, enquanto para $16(29,6 \%)$ a resposta foi melhorou muito. Dos 54 entrevistados, $4(7,4 \%)$ não responderam. Estes números podem ser observados na figura 4 .

Os acadêmicos foram perguntados quanto à capacidade de identificar a aplicação prática dos conceitos estudados após o curso de Informática Médica. Para $4 \quad(7,4 \%)$ entrevistados, a resposta foi de nunca identificar esses processos. A maior parte, 32 $(59,3 \%)$ acadêmicos, respondeu ter identificado alguns dos conceitos abordados, enquanto $2(3,7 \%)$ afirmaram ter identificado todos mas esquecido em algum momento. Para $12(22,2 \%)$ alunos, a resposta foi conseguir identificar todos e acreditar que ainda poderiam identificar estes conceitos. Dentre os participantes da pesquisa, $4(7,4 \%)$ não responderam.

Outros itens da pesquisa questionaram os alunos quanto ao acesso a computador com internet em seus domicílios e quanto ao uso deste como complemento das atividades acadêmicas de modo geral. Dentre os discentes, $50(92,6 \%)$ responderam SIM para estas questões, enquanto $4(7,4 \%)$ não responderam.

O último item do questionário perguntou aos participantes quanto à identificação no ambiente virtual de algum material voltado à promoção de saúde (campanhas temáticas como outubro rosa, novembro azul, setembro amarelo, entre outras). Para 2 grupos de 4 $(7,4 \%)$ alunos, a resposta foi não ter identificado este tipo de material e raramente ter identificado estes materiais, respectivamente. Para $23(42,6 \%)$ entrevistados, a resposta foi ter identificado este tipo de material com frequência, ao longo da disciplina, 19 $(35,2 \%)$ responderam ter identificado este tipo de material durante todo o curso, enquanto $4(7,4 \%)$ não responderam a este item. 


\section{Conclusões}

A proposta desta pesquisa, contextualizada neste artigo, é por meio da utilização de materiais audiovisuais com conteúdo voltado para a área da saúde (OVAs), propostos pelo professor da disciplina específica, desenvolver atividades ligadas à aprendizagem, promovendo o desenvolvimento do conhecimento como um processo espontâneo, ou seja, com auto regulação. Para isso, o sujeito tem que ser ativo, interagindo com o objeto e, consequentemente, defrontar-se com uma perturbação externa e interna, reagir como fim de compensar e tender para o equilíbrio como processo ativo. Este processo de equilibração toma forma de uma sucessão de níveis de equilíbrios, situação fundamental desenvolvida pela disciplina e os objetos de aprendizagem, ou seja, para Piaget ocorre o fator de equilibração e reequilibração.

Constata-se esta equilibração a partir da análise dos dados obtidos pela pesquisa realizada. Percebe-se que a ferramenta de avaliação, através do questionário presencial e online, permitiu às equipes de alunos uma revisão de seus trabalhos e a abertura para os outros alunos observarem e avaliarem seus modos de aprendizagem, comparando-os com os de outras equipes. Já ao professor e monitores da disciplina, a pesquisa possibilitou o acompanhamento, a orientação e a avaliação dos projetos de OVAs e/ou OVAs prontamente desenvolvidos pelos grupos.

Além disso, o método adotado na disciplina, com atividades online, individuais e em grupo, além das tradicionais atividades presenciais, traz uma proposta de avaliação contínua e progressiva. Dessa forma, todas as atividades realizadas nos projetos tiveram avaliações pontuais, feitas de forma individualizada.

\section{Referências}

AMEM BMV, Nunes LC. Tecnologias de informação e comunicação: contribuições para o processo interdisciplinar no ensino superior. Rev Bras Educ Med. 2006;30(3):171-80.

BASTOS FILHO, O. C.; AXT, M.; LABIDI, S.; SILVEIRA, P. D.; THOMAS, A. R. SISTEMA INTELIGENTE DE DESAFIOS ABERTOS IOCS: uma proposta de adaptação dos padrões do Método Clínico Piagetiano em Plataforma Multiagentes. In: Revista Novas Tecnologias na Educação, ISSN 1679-1916, 2006 b.

BASTOS FILHO, Othon de Carvalho. Sistema Inteligente de Desafios Abertos IOCS: uma proposta de adaptação dos padrões do Método Clínico Piagetiano utilizando provas lógicas de concepções abertas em Sistema Multiagentes / Othon de Carvalho Bastos Filho - Porto Alegre: PPGIE da UFRGS, 2006. 149f. : il.

Brasil. Ministério da Educação. Conselho Nacional de Educação. Câmara de Educação Superior. Parecer CNE/CES n. 1133, de 7 agosto de 2001. Institui as diretrizes curriculares nacionais dos cursos de graduação em enfermagem, medicina e 
nutrição. Diário Oficial da República Federativa do Brasil [online] Brasília (DF). 2001Out. Disponível em: http://www.mec.gov.br/Sesu/diretriz.shtm\#legislação.

CRESWELL, J. W. Projeto de Pesquisa: métodos qualitativos, quantitativos e misto. 3. Ed. Porto Alegre: Artmed, 2010.

MEURER, H.; SZABLUK, D. PROJETO E: Metodologia Projetual para Desenho de Ambientes Dígito-Virtuais. In: VAN DER LINDEN, J. C. S.; MARTINS, R. F. F (Orgs). Pelos Caminhos do Design. Rio de Janeiro: Editora Rio Books, 2012.

MOURSUND, D. El Aprendizaje por Proyectos Utilizando las Tecnologías de la Información y las Comunicaciones. Eduteka, 2004.

NICOLA, L. B.; RODRIGUES, A. P. Objetos de Aprendizagem como Potencializadores no Estudo da Biologia. Renote, Porto Alegre, v. 9, n. 1, p1-10 jul.

PIAGET, J.; et al. A Tomada da Consciência. Trad. Edson B. de Souza. São Paulo: Melhoramentos e EDUSP, 1977. 211p.

RIPPLE, R e ROCKCASTLE, V. Piaget rediscovered. Cornell University, 1964.

SALDANHA, Camila Teixeira. Interação dos Fóruns de Discussão: Uma análise Linguística. 2011. 201 f. Dissertação (Mestrado) - Curso de Pós-graduação em Educação, Universidade Federal de Santa Catarina, Florianópolis, 2011. 